\title{
Lidil
}

Revue de linguistique et de didactique des langues

$45 \mid 2012$

Pratiques de formation à la lecture-écriture des adultes en parcours d'insertion

\section{Interactions orales et réflexion sur la langue dans la réécriture chez l'adulte}

\section{Fatima Chnane-Davin et Claudine Lafarge}

\section{OpenEdition}

\section{Journals}

Édition électronique

URL : http://journals.openedition.org/lidil/3168

DOI : 10.4000/lidil.3168

ISSN : 1960-6052

Éditeur

UGA Éditions/Université Grenoble Alpes

Édition imprimée

Date de publication : 15 mai 2012

Pagination : 107-121

ISBN : 978-2-84310-226-4

ISSN : $1146-6480$

Référence électronique

Fatima Chnane-Davin et Claudine Lafarge, «Interactions orales et réflexion sur la langue dans la réécriture chez l'adulte », Lidil [En ligne], 45 | 2012, mis en ligne le 15 novembre 2013, consulté le 30 avril 2019. URL : http://journals.openedition.org/lidil/3168; DOI : 10.4000/lidil.3168

(C) Lidil 


\title{
Interactions orales et réflexion sur la langue dans la réécriture chez l'adulte
}

\author{
Fatima Chnane-Davin et Claudine Lafarge*
}

\begin{abstract}
RÉSUMÉ
Cette contribution sur les adultes en grande difficulté à l'écrit repose sur l'exploitation croisée de données écrites et vidéographiques recueillies durant une année de formation acquisition des savoirs fondamentaux (ASF) en atelier d'écriture-réécriture. On abordera particulièrement la question de l'interaction pendant la réflexion collective dans l'atelier de réécriture. On mettra l'accent sur le dispositif de la formatrice lors de la mise en commun des connaissances de chacun des stagiaires adultes. On examinera comment, par l'étayage, elle les amène à discuter différents points d'étude de la langue. Pour ce faire, ils échangent méconnaissances, connaissances, erreurs, doutes; ils négocient et s'impliquent peu à peu dans un travail coopératif qui s'instaure. On tentera de montrer à travers les résultats de cette recherche que l'atelier de réécriture des adultes permet la construction d'un espace d'expression validé par l'écoute des pairs, l'intégration de l'écrit dans la vie quotidienne dans le but de se construire des savoirs nouveaux. Les interactions entre les stagiaires et leur formatrice autour de savoirs linguistiques s'engagent et amènent un changement notable dans le rapport à l'écriture, parce que l'erreur dans les productions écrites est dédramatisée.
\end{abstract}

\section{ABSTRACT}

This contribution on the adults in trouble rests on the crossed exploitation of written and videographic data collected during one year of training ASF in workshop of writing-rewriting. What will be particularly approached is the co-reflection in the workshop of rewriting. We shall emphasize the device of the trainer during the pooling of the knowledge of each of the grown-up trainees. We shall examine how, by the propping up, it brings them to discuss various points of the study of the language. To do it, they exchange misunderstandings, knowledge, errors, doubts;

* Aix-Marseille Université (AMU), UMR P3 ADEF. 
they negotiate and get involved gradually in a cooperative work which is established. What we would like to show through the results of this research it is that the workshop of rewriting of the adults allows the construction of a space of expression validated by the listening of the peers, the integration of the writing in the everyday life with the aim of building themselves new knowledges. The interactions between the trainees and their trainer around linguistic knowledges bring a considerable change in the relationship with the writing, because the error in the written productions is taken the drama out.

Dans cette contribution à la réflexion sur les pratiques de formation à la lecture-écriture des adultes, nous nous appuierons sur une recherche sur des adultes en difficulté à l'écrit en formation en ASF (acquisition des savoirs fondamentaux). Cette formation proposée par le Centre national de la fonction publique territoriale aux agents territoriaux en grande difficulté avec l'écriture ${ }^{1}$ est essentiellement composée de trois ateliers d'étude de la langue : l'atelier de fabrication d'exercices de français, l'atelier d'écriture et l'atelier de réécriture. L'ensemble vise l'appropriation des processus rédactionnels.

L'analyse d'extraits de corpus choisis dans une recherche effectuée pendant la formation des adultes en ASF concerne la phase de réécriture de productions écrites par les stagiaires où l'accent est mis sur les interactions pendant la co-réflexion linguistique. Cette phase se caractérise par une dédramatisation de l'erreur (Marquillo-Larruy, 2003) par la formatrice face à des stagiaires qui opposent souvent des résistances à corriger leurs productions. Ces résistances s'enracinent en amont de l'apprentissage technique, dans les composantes du rapport au langage que sont les conditions de l'efficacité du travail scolaire, les attitudes conscientes/inconscientes envers l'écriture et les postures d'écriture. L'apprenant considère l'erreur, tout au moins au début comme sans importance et elle est «interprétée en termes de défaillance ce qui, parfois, va jusqu'à remettre en cause sa valeur en tant que personne » (p. 121). Ainsi, dans la formation des adultes en ASF, écriture, réécriture et réflexion collective orale sur la langue tiennent une place prépondérante en tant qu'outils de travail de la langue et de co-construction de la culture du groupe. L'apprentissage est, alors, le résultat d'une interaction sociale dans le sens de Vygotski (1997).

1. Blocage, absence de syntaxe, pauvreté de vocabulaire, non maitrise de l'orthographe, etc. 
On va examiner dans cette contribution comment la formatrice organise les conditions des interactions entre des adultes faiblement littéraciés lors d'une réflexion collective pendant la réécriture de passages de leurs propres productions. Notre objectif dans l'observation de cette classe est de comprendre comment le groupe se forge sa propre «culture » et change son rapport au langage oral et écrit pendant les interactions lors du retour sur les erreurs des uns et des autres. Nous mettrons l'accent sur la phase de mise en commun des connaissances des stagiaires adultes afin de comprendre si le fort étayage de la formatrice amène ces stagiaires à discuter et à résoudre différents points d'étude de la langue et à construire des savoirs nouveaux et combler des lacunes, particulièrement à l'écrit.

\section{Cadre théorique}

Notre cadre théorique sur les interactions lors de la co-construction des savoirs s'inscrit dans la théorie de l'action ${ }^{2}$ conjointe (Sensevy et Mercier, 2007) de la formatrice et des stagiaires adultes en formation. Il s'agit d'actions coordonnées par plusieurs interactants dans « un discours dialogal (produit par plusieurs locuteurs en chair et en os) » (KerbratOrecchioni, 2005 : 16) organisées par la formatrice. On expliquera comment ces interactants échangent méconnaissances, connaissances, erreurs, doutes et négocient en s'impliquant peu à peu dans un travail coopératif (Baudrit, 2005) qui s'instaure lors de la co-construction du discours. On s'appuiera également, dans notre analyse des interactions verbales, sur les travaux de Kerbrat-Orecchioni (1990) et d'Austin (1970) pour étudier une situation où il y a une production d'un acte locutoire qui a un sens et une référence où s'exerce un pouvoir illocutoire (j'instaure une relation) ou perlocutoire (j' agis, je cherche à agir sur l'interlocuteur) avec une intention. L'analyse de situation d'interaction n'est jamais facile « parce que, souvent enfouie sous les discours [l'interaction] ne s'offre que rarement à l'observateur-linguiste, qui doit la recomposer à partir de multiples traces qu'elle laisse en surface » (De Nuchèze, $1998: 5$ ).

2. «Par action, j'entends simplement le fait que les gens agissent » (Sensevy, dans Sensevy et Mercier, 2007 : 14). 


\section{Cadre de l'activité observée}

La formatrice observée met en place un dispositif de formation visant d'une part l'appropriation des processus experts de construction du texte et sa cohésion interne. D'autre part, le dispositif a pour but l'appropriation des processus d'accord (en genre et en nombre, sujet-verbe, participe passé avec avoir et être), de la conjugaison et de l'homophonie. À cette fin, elle relie le travail de trois ateliers : l'atelier de fabrication d'exercices, l'atelier d'écriture et l'atelier de réécriture. Dans les deux premiers ateliers, les stagiaires dépassent le blocage de l'expression écrite; ils produisent des textes dans lesquels ils se sont investis, qu'ils ont correctement construit avec des actions situées dans le temps et dans l'espace grâce aux connecteurs logiques. Malgré cette évolution dans l'écriture, la phase de réécriture reste une épreuve en soi. C'est cette phase qui nous intéresse ici.

Dans les séances observées pendant une période de formation de novembre à mai, l'objectif visé par la formatrice est d'amener les stagiaires à s'approprier des processus de mise en lien des différents éléments de la phrase pour effectuer les accords correctement et s'approprier des processus de remaniement de la phrase, lequel comprend trois étapes : remaniement, relecture, réajustement du tronçon modifié au sein du texte. Cet atelier repose sur la réécriture d'un extrait de production réalisé lors d'un atelier d'écriture antérieur. C'est un atelier volontairement déconnecté de la phase de production qui dure environ trois quarts d'heure. La formatrice inscrit sur le tableau blanc l'extrait d'un texte produit par l'un des stagiaires lors d'un précédent atelier d'écriture. Elle prend soin de choisir des textes contenant des erreurs en rapport avec la progression pédagogique développée en atelier de fabrication d'exercices. Ce moment sensible de réécriture de passages, pendant la réflexion linguistique et la correction d'erreur, peut facilement réactiver les processus d'inhibition de l'apprenant. En effet, le fait de voir son texte au tableau durant cette séance de travail et le nombre d'erreurs à travailler par le groupe peuvent être décourageants si l'apprenant n'est pas pleinement acteur de cette réécriture (Chabanne et Bucheton, 2000). C'est pour cela que la formatrice prend soin de valoriser les interventions de l'auteur sur cet écrit intermédiaire. Pendant cette activité de réécriture, la concertation et la réflexion sur la langue se mettent en place et amènent un changement notable dans le rapport à l'écriture. L'analyse de la séance filmée montre que les stagiaires questionnent, demandent des éclaircissements et entrent dans la co-construction de la résolution des problèmes puis des savoirs. 
Une fois l'extrait inscrit au tableau, rapidement les stagiaires s'organisent, choisissent qui va écrire au tableau, repèrent les différents feutres à utiliser, une couleur différente pour chaque passage de réécriture. Ils démarrent la tâche en moyenne 30 secondes après la fin de l'inscription du texte au tableau. Dans un travail collectif et collaboratif, ils corrigent l'extrait. Cette réécriture se fait toujours au fil du texte dans un échange sur les différentes séquences à réécrire. «Les participants coordonnent leurs activités pour produire en commun cet objet final » (Kerbrat-Orecchioni, $2005: 20)$. Une fois le travail de groupe achevé, la formatrice intervient et signale le nombre d'erreurs qui subsistent encore dans le texte pour initier une nouvelle phase d'interaction et de réécriture. Une action qui peut être répétée plusieurs fois jusqu'à ce que le groupe ait repéré et travaillé toutes les erreurs présentes. Les interventions de la formatrice sont courantes en début de dispositif, et deviennent de plus en plus sporadiques pour disparaitre quasiment en fin de parcours. Il s'agit, en général, d'un « discours de guidance de l'observation, d'aide à l'émergence d'une notion et d'un concept » (Verdelhan, 2002: 96).

Le travail d'échange verbal autour de la réécriture du texte, tâche ardue pour les stagiaires, demande une organisation et surtout des encouragements de la part de la formatrice. Avant de comprendre l'enjeu de cette activité, certains stagiaires, notamment les plus en difficulté, éludaient cette étape pourtant indispensable en exprimant leur incapacité à voir les erreurs : « ah, mais de toute façon, je ne les vois pas, pour moi y en a pas », une façon de « dénier » l'erreur (Astolfi, 1997). Parfois, ils vivaient mal d'être dans le doute perpétuel : « je corrige une première fois et après $\mathrm{j}$ 'ai le doute et je recorrige et je peux corriger encore une fois après ça, alors là, ça m'énerve ».

\section{Organisation de la réflexion collective sur la langue dans les textes écrits}

Il s'agit dans cette partie de rendre compte de la manière d'installer une réflexion collective favorable à l'appropriation des processus d'étude de la langue. La formatrice a ici fait le choix du texte de Franck, un des stagiaires, qui sera retravaillé collectivement parce qu'il présente matière à réflexion au regard de l'apprentissage qu'elle souhaite initier : le remaniement syntaxique de la phrase. 
Le choix porte sur le texte suivant (avant réécriture) :

Nous avons visité les Baux de provençe. perdu sur un plateaux sur-tlombant la végétation, un chateau Médievale entouret d'un village d'époque Nous avons vu une reconstitution d'une époque Médiévale des cavalier avec leurs cuirase des villageois. il y avait un compteur des chanteurs a compagner de musicien on se croyaient projetaient dans le passé.

Outre les erreurs relatives à la norme élémentaire qui consiste ici à savoir faire une phrase comprenant un sujet, un verbe et un complément, cet extrait a besoin d'être restructuré pour recouvrer toute sa cohérence. Il s'agit de résoudre des problèmes morphosyntaxiques, lexicaux et orthographiques dans le texte (des accords, des verbes conjugués, des marques de ponctuation, etc.). Quant aux idées, elles sont pertinentes mais agglutinées les unes aux autres. La réécriture de cet extrait constitue un milieu, dans le sens d'un espace didactique où sont organisées les conditions d'apprentissage pour susciter des conflits sociocognitifs (Vygotski, 1997) dans l'accommodation à un savoir nouveau. Le travail de réécriture ${ }^{3}$ par les stagiaires à partir du texte écrit par la formatrice au tableau est filmé et se présente sous la forme suivante :

Nous avons visité les-Baux de provenȩe . perdu dans la campagne sur le village des provence. Perdu

un plateaux sur-tlombant lavégétation, un ehateatt Médievale entouret plateau surplombant château médiéval entotré fort domine le

d'un village d'époque Nous avons vu une reconstitution d'une époque

Médiévale des cavaliers avec tetres etrirase des villageois it y avait un médiévale leur cuirasse, . Il

conteur des chanteurs a eompagner de musicien ont se eroyaient accompagnés musiciens. On croyait progetaient dans le passé.

projeté.

Les stagiaires, Franck (F), Jean-Luc (JL), Jean-Claude (JC), Évelyne (E) et Mireille (M) accompagnés de la formatrice $(\mathrm{CL})$ participent à cette séance de travail vidéographiée. Que s'est-il passé pendant ces corrections en termes d'interactions verbales autour d'un texte écrit?

3. En gras les corrections apportées. 
Lors de l'étude des interventions de la formatrice, on a constaté que cette dernière attend des stagiaires qu'ils mettent en commun leurs savoirs pour résoudre une difficulté. Elle attend d'eux qu'ils s'écoutent les uns les autres. De fait, systématiquement, elle reprend l'intervention passée sous silence. Elle attend également qu'ils prennent le risque d'auto-valider leurs savoirs, parfois elle les valide elle-même. Elle refuse de valider de manière explicite, surtout quand elle est sure que le savoir est intégré au contexte cognitif commun :

$M$ : plateau y a pas de $x /$ c'est quand y en a plusieurs/

$E: y$ a pas de $x /$ hein /

$J C: y$ a pas de $x /$

$C L$ : voyez entre vous/

Les participants se retrouvent dans une réflexion linguistique qu'ils effectuent conjointement mais des accords ne semblent pas s'établir entre eux. La formatrice accompagne le groupe dans la découverte d'un accord connu dans une situation nouvelle. Par exemple, lors de la réécriture du passage relatif à l'accord de « château médiéval », initialement écrit « chateau Médiévale » par Franck, elle fournit l'étayage nécessaire parce qu'elle sait que cette situation ne fait pas partie du contexte cognitif commun. Elle intervient également pour réguler le temps didactique. À la fin de la première étape de cet atelier, le groupe se dédouble et certains avancent, alors que la question de l'accord sujet-verbe n'est pas encore entièrement résolue dans le passage « on se croyaient progetaient dans le passé ».

CL: n'allez pas trop vitel hein / d'abord vous vous occupez de la terminaison de croyait et après vous vous occuperez du sel

La formatrice peut solliciter le groupe pour rappeler un savoir connu, employé dans une situation nouvelle pour convaincre le groupe et l'orienter vers la solution :

$J L$ : le deuxième verbe, i passe pas à l'infinitif।

JC : ouil ( 2') c'est sûr que ont /c'est pas çal

$J L:$ moi lje le mettrais à l'infinitif

$J C$ : je crois qu'il a raison/ derrière un verbe c'est l'infinitif

$J L:$ (inaudible)

$C L$ : essayez mordre et mordu I

$J C$ : si on essaie çal c'est é 


\section{Interactions et ratés du « jeu 4 » de langage}

Une rupture dans le contrat didactique initial intervient à la $25^{\mathrm{e}}$ minute de la séance. Les stagiaires ont l'impression d'avoir achevé la tâche de réécriture alors qu'ils ont résolu uniquement tout ce qui relevait de la norme élémentaire définie auparavant. La formatrice les invite alors à prendre en charge un élément qui relève de la norme complexe, permettant l'organisation et la cohérence de la phrase, du texte et du discours, et le remaniement de la phrase dans l'unité du texte. Elle relit le début du texte et attire l'attention du groupe sur l'absence de verbe de la deuxième phrase en posant des questions au groupe.

$C L$ : (relit le texte à haute voix, reprend) nous avons visité les baux de Provence $\backslash$ perdu sur un plateau surplombant la végétation un château entouré d'un village d'époque $\backslash$ alors/ où est le verbe I

$F$ : (inaudible) entouré /

JC : oui entouré /

$J L$ : (Il relit) un château médiéval / entouré c'est le verbe I

CL : i faut qu'il soit conjugué I

Les partenaires de l'interaction arrivent à une organisation pour faire avancer la réflexion linguistique où chaque locuteur est soit initiateur soit continuateur de l'interaction autour du même objet. Une gestion des tours de parole se met en place pour annoncer la suite et repérer l'objectif du « jeu » de langage : repérer et conjuguer un verbe dans une phrase. Mais les difficultés des stagiaires peuvent freiner et même ralentir voire rompre l'interaction :

$C L$ : alors nous avons visité les baux de provence $\backslash$ perdu sur un plateau surpomblant/ surplombant pardon la végétation (5') qu'est que c'est/ qui est perdu sur un plateau ।

Jean-Luc est le seul à répondre. Le reste du groupe demeure silencieux scrutant le texte inscrit au tableau. Le silence s'installe quelques secondes. Puis chacun reprend la parole à tour de rôle en suivant la réflexion engagée. Une discussion de groupe démarre à propos de l'ajout de « village ». Mireille se lève pour reprendre sa place de « scribe » au tableau. Cette scène a duré près de quatre minutes. La reprise de la

4. «Jeu» en tant qu'action didactique où sont mises en œuvre des stratégies d'apprentissage. 
discussion et le retour de Mireille au tableau marque, selon nous, l'adhésion du groupe au nouveau jeu.

Les règles de fonctionnement ont changé, le questionnement est amené par la formatrice et sera maintenu jusqu'à la fin de la séance. Plutôt que d'énoncer la nouvelle tâche à réaliser au groupe, elle a fait le choix de montrer celle-ci dans sa spécificité et sa complexité.

\section{Régulation des interventions}

La formatrice influe au plus près sur la production verbale des stagiaires et favorise la co-construction des interventions sans se substituer cependant à eux. Elle verbalise la procédure à adapter et renvoie ensuite au groupe les questions qui émergent :

CL: (inaudible) / il faut l'ajouter que c'est un village

$J C$ : à quel endroit /

CL : heu / où / où vous le mettriez /

$J L:$ nous avons visité un vi-[llage

JC : [après baux de provencel nous avons visité les baux de provencel un village perdu /

On note que ce procédé stimule la réflexion des stagiaires :

$C L$ : ça revient au même mais /il faut : un verbe conjugué dans cette phrasel i peut pas y avoir de phrase sans verbe conjugué dans ce cadre-là

$J L$ : en fait/ c'est au départ que

$E$ : il était perdu sur un plateau /

$C L$ : perdu sur un plateaul

$E$ : on peut pas dire il était perdul

Les stagiaires commencent à prendre la mesure de la nouvelle tâche à entreprendre et du nouveau savoir à mettre en œuvre. La formatrice expose la multiplicité de choix qui s'offre à eux au regard du lien entre syntaxe et sémantique et des effets que ce choix peut produire sur le texte, en entrainant notamment la nécessité d'un prochain remaniement de phrase, en fonction de ce que l'auteur a voulu exprimer.

$E$ : on peut pas dire il était perdu/

$C L$ : ouil on peut dire il était perdu sur un plateau surplombant la végétation/ à ce compte là on met à nouveau un point après végétation $\backslash$ et il nous faut une autre phrase avec un château médiéval

$E$ : oh pétard (rires du groupe) 
CL : eh/ oui / il avait trois quatre idées qui étaient complètement compressées en une seule phrase et ça ça va pas \à l'écrit c'est pas possible $\backslash$ à l'oral / on va comprendre

JC : ouais

Évelyne prend pleinement conscience de la procédure à adopter pour faire face à la difficulté syntaxique rencontrée. Sa réaction quant à la complexité de la tâche en témoigne. Jean-Luc lance un « ouais » qui semble encore dubitatif. Il doute, semble-t-il, de ses capacités à y faire face. Ce « ouais » est « un simple régulateur » (Kerbrat-Orecchioni, 2005 : 38) et représente une forme de demande de confirmation.

Cette co-construction du discours par un travail collaboratif (Schegloff, 1982) montre l'avancée dans l'orientation de la réécriture vers la syntaxe et la cohérence sémantique des phrases dans le texte. Elle remporte l'adhésion des stagiaires grâce, certainement, à la combinaison de deux facteurs, d'une part l'adhésion à la nouvelle modalité relative à la part plus active de la formatrice dans la tâche; en effet, la syntaxe n'étant pas encore acquise par le groupe, un travail en autonomie n'aurait sans doute pas reçu le même accueil. D'autre part il semble que les stagiaires soient accoutumés à ce type d'évolution du contrat didactique, le peu de temps d'adaptation que cela a nécessité, la reprise tacite du fonctionnement groupal intégrant la présence active de la formatrice en témoigne.

\section{Co-élaboration du contenu de l'interaction}

On voit comment le contenu de l'interaction se trouve co-élaboré (création de contenu) par la formatrice et les stagiaires dans ces actions conjointes dans le «passage de la cédille de Provence » puis dans le passage de la construction de la phrase « perdu sur un plateaux sur-tlombant».

Lors de l'étude du passage de la cédille (voir texte de Frank), des conflits et des négociations relationnels et cognitifs ont eu lieu. Dès la première minute, une discussion interstagiaire s'engage autour de la nécessité de mettre une cédille ou pas à « provence ». Évelyne affiche fermement son opposition à Franck lequel demande des explications. Évelyne donne une réponse qui ne convainc pas Franck. Du coup, Mireille commence à chercher dans ses savoirs des éléments de justification.

E : elle y est jamais /je sais pas/ je l'ai jamais vu écrite avec une cédille $\backslash$ moil provence /

$F$ : si tu enlèves la cédille ça fait $[k]$

$M$ : pro\ven \çalel y a la cédille 
E : provençal/ ouil

$J C$ : provençalloui

E : mais provence $\backslash$ non/ j'ai jamais vu moi

$M$ : aix en provence non/ il y est pas /

Jean-Claude sera alors convaincu et se dira contre l'emploi de cette cédille. Franck trouve une partie du savoir exploitable ici et Jean-Luc apporte un complément d'information.

$F:$ sinon $\backslash$ ça fait le son/ $[k] /$ et si on lève la cédille ça fait

$C L$ : c'est çal le trucl à quoi sert la cédille

$J L:$ faut déterminer à quoi elle sert [quand on sait à quoi sert la cédille/

$E$ : peut-être par rapport au

JL : alors/ à quoi elle sert/ la cédille / à [se] comme dans maçon si tu

lèves la cédille [ça fait macon

E : eh ben oui

$F$ : ça fait macon

À ce stade Jean-Luc et Franck ne se sont pas encore rendu compte que leur assertion joue en faveur de ce qu'amène Évelyne.

$M$ : provençal/

E : ouais mais y a peut-être un truc

$C L$ : Mireille nous dit à provençal il y est

Ici, la formatrice est intervenue pour attirer l'attention du groupe sur le fait que ce qu'amène Mireille complète ce qui vient d'être dit par Franck et Jean-Luc. Puis, elle insiste pour que la question de Jean-Luc soit prise en compte :

$C L$ : mais répondez à la question de JL /à quoi sert la cédillel

$J C$ : eh bè c'est pour pas que ça fassel [k]

$J L:$ et là I

CL : et là /

L: si on

E : je pense pas qu'en plus le ce à la fin ça se dit ce c'est pas besoin de\ heu y a pas la cédille parce que proven \ce /ce à la fin i ce dit ce

$F:$ donc y a pas la cédille (dans sa barbe)

Franck vient de comprendre. Il a rassemblé les pièces du puzzle, en l'absence de cédille le « $\mathrm{c}$ » produit le son [k] devant le «a » et le « $\mathrm{o} »$, et devant un «e » le « ce » se prononce [s] donc elle est inutile. Il sait désormais que «Provence» ne prend pas de cédille. Reste à convaincre Jean-Luc qui lui n'a pas suivi la même réflexion. Il semble bloqué, tout fier qu'il est d'avoir trouvé un élément décisif de stratégie. 
Il semble plus intéressé par le fait d'avoir raison, de gagner dans le jeu de la négociation que dans le jeu d'apprentissage. C'est Jean-Claude qui va apporter le complément d'information qui, ajouté à ce qui précède, servira de preuve à l'assertion d'Évelyne.

$$
\begin{aligned}
& J C: \text { c'est comme si tu disais/ tracel ou : } \\
& E: \text { voilàl } \\
& J C: \text { ou face } \\
& E: \text { ou menace } \\
& F: \text { oui / mais t'à l'heure regarde } \\
& J C: \text { non } \backslash \text { Provence i faut pas de cédille } \\
& E: \text { et provença\ ouil }
\end{aligned}
$$

Jusqu'à ce stade, l'intervention de la formatrice a été propice à Franck pour l'avancée dans la découverte du savoir, mais pour Jean-Luc, c'est l'inverse qui s'est produit. Les deux interventions de la formatrice qui ont validé sa question «à quoi sert la cédille/ » l'ont, en fait, maintenu inutilement dans le rapport de force au détriment de l'avancée dans la réflexion.

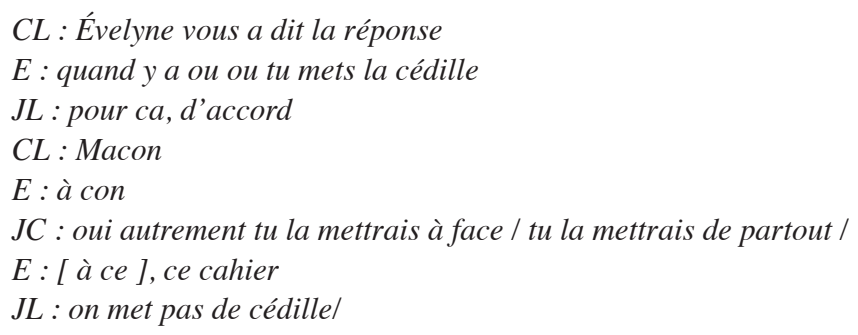

En guise de synthèse, la formatrice redonne la parole à Évelyne qui résume avec l'appui de Jean-Claude, l'essentiel de la règle relative à l'emploi de la cédille en orthographe. C'est ce qui va permettre à Jean-Luc d'accéder à la compréhension de son erreur et autorisera son adhésion à l'opinion du reste du groupe. À noter le rôle d'Évelyne, ses acquis permettent de faire avancer la co-construction, qu'elle ponctue de validations. Elle ne parvient pas, seule, en cours de travail, à produire l'explication idoine, par contre, elle sait la reconnaitre quand elle émerge, élément par élément. 


\section{Interactions, modalités du travail et situation topogénétique}

Comme on l'a constaté, depuis le début de ces échanges et selon les modalités mises en place, on a assisté à « une négociation des places interactionnelles (souligné par l'auteur) » (Kerbrat-Orecchioni, 2005 : 109). Nous avons observé dans le corpus les pronoms personnels, lesquels caractérisent les modalités du travail et permettent d'attribuer à chacun sa place lors de l'interaction, par exemple :

- On marque une fusion langagière entre la formatrice et les stagiaires pour ne constituer qu'un seul groupe. L'objectif d'une telle pratique verbale, marque d'une forme d'ignorance générale, est de faire avancer le groupe ensemble. Cette situation « d'ignorance fictive », où la formatrice fait semblant d'être dans la même situation que le stagiaire, légitime l'ignorance de ce dernier afin de chercher ensemble la solution à un problème donné, parfois « au prix d'un incessant travail de rafistolage (un bricolage interactif) que les interactants parviennent à construire ensemble » (KerbratOrecchioni, 2005 : 109). C'était le cas lors du débat autour de la « cédille».

- Tu/vous (+ ton//votre/vos) marquent une opposition entre le groupe et le stagiaire ou le groupe qui produit la stratégie. Le discours injonctif est alors très utilisé et la réponse ou l'accomplissement d'une tâche est attendue de la part de la ou des personnes à qui s'adresse l'injonction.

Ces modes d'interaction portés par le discours de l'enseignant (Chnane-Davin et Cuq, 2008), ici une formatrice, en classe ne sont pas inédits pour l'adulte apprenant qui a été scolarisé quand il était jeune. Ce qui change, et ce qui est nouveau pour certains, c'est l'implication dans son propre apprentissage et la co-construction fondée sur des interactions langagières. C'est pour cela qu'on peut dire que, si le discours de la formatrice dans la formation ASF est nouveau pour l'apprenant, la situation topogénétique spécifiée est également nouvelle. Souvent ces stagiaires adultes sont passés, quand ils étaient élèves, par un système scolaire traditionnel où la place de l'enseignant et celle de l'élève ne changent que rarement. Le discours est marqué par je-tu, rarement par « on »/« nous » prédominé par l'absence de situations de recherche commune. 


\section{Éléments de conclusion}

« À quoi ça va nous servir de connaitre toutes ces fonctions? ";

"Ils auraient pas pu faire plus simple, l'accord du participe passé? »;

"la langue française, c'est vraiment très compliqué ».

C'est ainsi que parlaient ces adultes au début de la formation ASF et avant de comprendre le sens des apprentissages linguistiques et leur utilité dans toute situation d'écrit grâce à des interactions verbales les impliquant dans leur propre apprentissage. Ces interactions changent, en effet, leur rapport au langage oral et les font avancer dans l'appropriation du langage écrit. Comme on l'a constaté tout au long de l'analyse de ces interactions autour d'objets linguistiques, les accompagner à entrer dans la littéracie et à améliorer leurs écrits, c'est les aider à s'engager dans une réflexion de co-construction de savoirs nouveaux à partir de leurs propres productions. Cette façon de faire leur permet de comprendre le fonctionnement, ici, des objets linguistiques dans une phrase ou dans un texte. Cette réflexion collective orale dans une action conjointe sur la langue met ces adultes dans des conflits socio-cognitifs (Vygotski, 1997) où l'interaction interpersonnelle s'effectue dans un milieu didactique aménagé. Dans ce milieu, le discours de la formatrice, qui est pour l'essentiel un discours d'étayage (Bruner, 1986), et les interactions entre pairs permettent aux stagiaires de comprendre la complexité de la tâche (l'exemple d'Évelyne) et d'arriver à bout de problèmes qu'ils n'auraient pas pu résoudre seuls. Notons qu'au cours des interactions, certains éléments du cadre communicatif sont restés stables (Kerbrat-Orecchioni, 2005), d'autres ont changé pendant les échanges. Des négociations, des oppositions (notamment autour de la cédille), ont fait avancer à la fois la co-construction des savoirs des objets linguistiques et leur intériorisation.

\section{RÉFÉRENCES BIBLIOGRAPHIQUES}

Astolfi J.-P. (1997) : L'erreur, un outil pour enseigner, ESF éditeur, coll. «Pratiques et enjeux pédagogiques ».

Austin L. L. (1970) : Quand dire c'est faire, introduction, traduction et commentaire par G. Lane, Paris, Seuil.

BAUDRIT A. (2005) : L'apprentissage coopératif, origine et évolution d'une méthode pédagogique, Bruxelles, De Boeck Université.

Bruner J. (1986-2008) : Culture et modes de pensées, Paris, Retz. 
Chabanne J.-C. et Bucheton D. (2000) : « Les écrits intermédiaires », $L a$ Lettre de la DFLM, n 26, 2000-1, juin 2000, p. 23-27.

Chnane-Davin F. et CuQ J.-P. (coord.) (2008) : Du Discours de l'enseignant aux pratiques de l'apprenant. Le Français dans le Monde, Recherches et applications, $\mathrm{n}^{\circ} 44$, juillet 2008, CLE International.

De Nucheze V. (1998) : Sous les discours, l'interaction, Paris, L'Harmattan. Kerbrat-Orecchioni C. (1990) : Les interactions verbales, Paris, Armand Colin.

Kerbrat-Orecchioni C. (2005) : Le discours en interaction, Paris, Armand Colin.

MARquIllo-Larruy M. (2003) : Interprétation de l'erreur, Paris, CLE International.

Schegloff E.A. (1982) : «Discourse as an interactional achievement: some uses of 'uhhuh' and other things that come between sentences », dans D. Tannen (éd.), Analyzing discourse: Text and Talk, Washington (DC), Georgetown University Press, p. 71-93.

Sensevy G. et Mercier A. (2007) : Agir ensemble, l'action conjointe du professeur et des élèves, Rennes, Presse universitaire de Rennes.

Verdelhan-Bourgade M. (2002) : Le français de scolarisation, pour une didactique réaliste, Paris, Presses universitaires de France.

Vygotski L. (1933) : Pensée et langage (traduction de F. Sève, avec des «Commentaires sur les remarques critiques de Vygotski » de Jean Piaget), Paris, Éditions Sociales, coll. «Terrains », 1985; rééditions : Paris, La Dispute, 1997. 
